\title{
REPRODUÇÃO HUMANA ASSISTIDA NO BRASIL: UMA ANÁLISE EPIDEMIOLÓGICA
}

\author{
ASSISTED HUMAN REPRODUCTION IN BRAZIL: AN EPIDEMIOLOGICAL ANALYSIS
}

DOI: 10.16891/2317-434X.v8.e1.a2020.pp437-444

Recebido em: 12.11.2019 / Aceito em: 09.03.2020.

\section{Yasmim de Alencar Grangeiroa, Pedro Walisson Gomes Feitosab ${ }^{b}$ Pedro Henrique Matos Grangeiro Cruz $^{b}$, Simone Pereira Matosa, Bruna Soares de Almeida ${ }^{a}$}

\author{
Centro Univertário Doutor Leão Sampaio \\ Universidade Federal do Caririb \\ *E-mail: yasmimdealencar2@live.com
}

\section{RESUMO}

A reprodução humana assistida (RHA) é definida como o conjunto de procedimentos que objetivam remediar a infertilidade humana. As técnicas mais evidenciadas na rotina são a inseminação artificial, a fertilização in vitro e a injeção intracitoplasmática de espermatozoides. As metodologias referidas possuem custo diversificado variando de acordo com grau de complexidade de cada uma. No Brasil, o Conselho Federal de Medicina é responsável por orientar a realização da RHA através de parâmetros não legislativos. Já a fiscalização das células reprodutivas manipuladas em laboratório é feita, anualmente, pelo Sistema Nacional de Produção de Embriões (SisEmbrio), criado pela ANVISA. O estudo objetivou retratar o cenário da RHA no Brasil, de acordo com o SisEmbrio, estimando de centros reprodutivos cadastrados no sistema e quantificando os oócitos produzidos, armazenados e descartados no ano de 2016. Trata-se de uma pesquisa de caráter transversal, retrospectivo, descritivo e quantitativo. A coleta dos dados demonstrou que $79 \%$ do material reprodutivo criopreservado no país localiza-se nas regiões Sul e Sudeste. Além disso, mais de $45 \%$ dos oócitos produzidos no Brasil, no ano de 2016, foram produzidos no estado de São Paulo que apresentou o maior índice nacional de reprodução humana assistida, seguido apenas pelos estados de Minas Gerais e do Rio de Janeiro, que produziram $10 \%$ e $9 \%$ dos oócitos, respectivamente. Dessa forma, constatou-se que, a busca pela RHA aumentou entre os anos de 2015 e 2017. Ademais, as regiões Sul e Sudeste assumiram liderança na realização das metodologias, pois, tais localidades possuem o índice econômico compatível com a realização das mesmas.

Palavras-chave: Criopreservação; Técnicas de Reprodução Assistida.

\section{ABSTRACT}

Assisted human reproduction (AHR) is defined as the set of procedures that aim to remedy human infertility. The techniques most evident in the routine are artificial insemination, in vitro fertilization and intracytoplasmic sperm injection. The referred methodologies have diversified cost varying according to the degree of complexity of each one. In Brazil, the Federal Council of Medicine is responsible for guiding the realization of the AHR through nonlegislative parameters. The supervision of reproductive cells manipulated in the laboratory is provided out annually by the National Embryo Production System (SisEmbrio), created by ANVISA. The study is aimed to portray the scenario of AHR in Brazil, according to SisEmbrio, estimating reproductive centers registered in the system and quantifying the oocytes produced, stored and discarded in 2016 . This is a cross-sectional, retrospective, descriptive and quantitative research. Data collection demonstrated that $79 \%$ of the cryopreserved reproductive material in the country lay in the South and Southeast regions. In addition, over $45 \%$ of oocytes produced in Brazil, in 2016 , were produced in the state of São Paulo, which had the highest national index of assisted human reproduction procedures, followed only by the states of Minas Gerais and Rio de Janeiro, which produced $10 \%$ and $9 \%$ of oocytes, respectively. Therefore, it was found that the search for AHR increased between the years 2015 and 2017. In addition, the South and Southeast regions assumed leadership in the implementation of the methodologies, as such locations have an economic index compatible with their realization.

Keywords: Cryopreservation; Assisted Reproduction Techniques. 


\section{INTRODUÇÃO}

A descoberta das células reprodutivas humanas, no início do século XX, despertou o interesse de diversos pesquisadores pela manipulação in vitro de gametas. Assim, no fim da década de 70, nasce o primeiro bebê de proveta do mundo. A reprodução assistida engloba um conjunto de técnicas e procedimentos que visam remediar a infertilidade humana (CORRÊA; LOYOLA, 2015). Para Matias (2018) as técnicas de Reprodução Humana Assistida são metodologias amplamente divulgadas pela mídia e frequentemente indicadas pela comunidade médica. Essa disseminação da temática, bem como, a necessidade da sociedade por tais tratamentos, fez com que surgissem cada vez mais estudos e pesquisas gerando, consequentemente, um maior desenvolvimento de técnicas e artifícios utilizados na RHA.

Nesse viés, surgem diversas metodologias para condução desses quadros, sendo a inseminação artificial, a fertilização in vitro e a injeção intracitoplasmática de espermatozoides as técnicas mais evidenciadas na rotina. $O$ custo dos procedimentos varia de acordo com o grau de complexidade de cada um e a técnica é definida consoante o caso apresentado (MESQUITA et al., 2016).

As razões que levam à procura dos centros reprodutivos são diversas e variam desde o desejo de gerar um filho sem parceiro conjugal, até o tratamento de enfermidades reprodutivas (BATISTA; BRETONES; DE ALMEIDA, 2016). As mulheres que buscam esse serviço por questões fisiológicas geralmente apresentam adversidades como endometriose, ovário policístico, miomas, pólipos, entre outros. Quando a infertilidade decorre de um problema físico masculino as causas mais comumente encontradas são varicocele, falhas genéticas, criptorquidia, presença de antígenos contra os espermatozoides, entre outros (LIMA; LOURENÇO, 2016).

Por se tratar de um problema de saúde pública, a regulamentação da Reprodução Humana Assistida (RHA), no Brasil, é realizada pelo Conselho Federal de Medicina (CFM), órgão que norteia a realização destes procedimentos através da instituição de parâmetros de caráter não legislativo. Além disso, para que se realizem os serviços da RHA, é necessário que os profissionais e instituições responsáveis obedeçam às legislações de biossegurança estabelecidas nacionalmente para 0 manuseio de materiais biológicos humanos e organismos geneticamente modificados (OLIVEIRA et al., 2018; SANTOS, 2019).
Nesse ínterim, a fiscalização das células reprodutivas manipuladas em laboratório no Brasil é feita anualmente, pelo Sistema Nacional de Produção de Embriões (SisEmbrio), criado pela ANVISA com o objetivo de monitorar a quantidade de embriões produzidos, armazenados e congelados no país (BRASIL, 2017b). Dessa forma, se faz necessário o cadastro anual das clínicas que ofertam a RHA, sendo penalizadas em infração sanitária quando não cadastradas no sistema nacional. Não obstante o SisEmbrio ser considerado uma fonte confiável de informação, entende-se os dados armazenados não condizem inteiramente com a realidade, visto que, apenas algumas das clínicas existentes estão cadastradas no sistema (MACHADO, 2016; NETO et al., 2018).

Portanto, agrupar dados quanto este tema permite que se conheça mais profundamente a situação da reprodução assistida no Brasil, garantindo o acesso descomplicado a informações, como quantidade de material reprodutivo armazenado e quantidade de reproduções assistidas realizadas anualmente por região ou estado. Destarte, a organização dos dados obtidos serve como fonte de pesquisa para aqueles que buscam entendimento aprofundado sobre a proposta. Diante do exposto, o presente estudo objetivou retratar o cenário da reprodução humana assistida no Brasil, de acordo com o Sistema Nacional de Produção de Embriões (SisEmbrio), estimando de centros reprodutivos cadastrados no sistema e quantificando os oócitos produzidos, armazenados e descartados no ano de 2016.

\section{MATERIAIS E MÉTODOS}

O estudo de caráter transversal e retrospectivo foi realizado na cidade de Juazeiro do Norte, no estado do Ceará no ano de 2019. Utilizou-se como fonte de dados o relatório anual do Sistema Nacional da Produção de Embriões (SisEmbrio) da Agência de Vigilância Sanitária (ANVISA), disponível no portal ANVISA (portal.anvisa.gov.br), sistema nacional que integra notificações de todos os centros de RHA no país. Ainda, foram selecionados artigos publicados entre 2014-2019, disponíveis nas bases de dados MEDLINE e SCOPUS, sendo utilizadas as palavras-chaves (MeSH) "Cryopreservation" e "Assisted Reproduction Techniques".

Os centros reprodutivos no Brasil foram categorizados por sua localização. A seguir, foi definida a quantificação do material reprodutivo coletado e armazenado nos anos de 2015 a 2017. Enfatizou-se a criopreservação de células-tronco embrionárias e tecidos germinativos, especificando a totalidade de 
material colhido, produzido e armazenado em cada estado do país. Após a coleta, os dados foram tabulados em planilhas do programa Microsoft Excel ${ }^{\circledR}$ e organizados em gráficos e tabela. Para construção de mapas, utilizou-se o portal online Map in Seconds. Posteriormente, os dados foram analisados e discutidos consoante a literatura evidenciada nas buscas digitais.

\section{RESULTADOS E DISCUSSÃO}

Figura 1. Centros de reprodução humana assistida existentes no Brasil e cadastrados no SisEmbrio, divididos por estado, no ano de 2016.

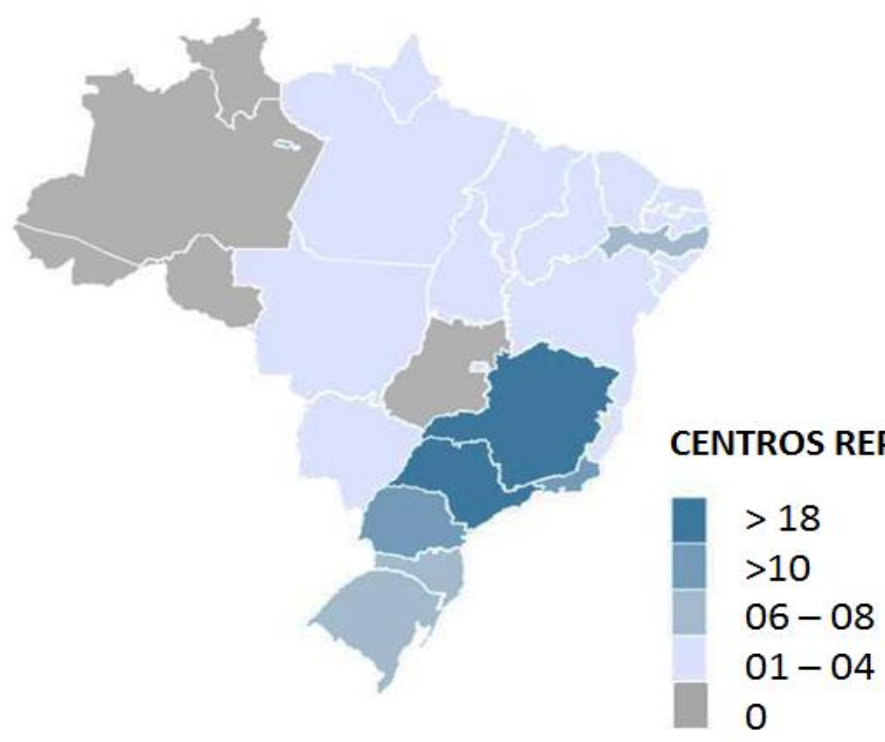

Fonte: (SISEMBRIO, 2016).

De acordo com Marciano et al., (2019), a realização da reprodução humana assistida (RHA) está ligada intrinsecamente ao desenvolvimento econômico da região. Uma vez que, para sua realização, são necessários investimentos na qualificação da mão de obra e na infraestrutura dos centros reprodutivos. Ainda, para a efetivação de um serviço qualificado em RHA, é necessário analisar a complexidade envolvida na temática da infertilidade que implica a carência de intervenções que incluam as dimensões emocionais e que considerem o contexto ambiental no qual a mesma está inserida (GASDA, 2015). Dessa forma, entende-se que o tratamento deve abranger muito mais que enfermidades, mas tratar sujeitos, escutando seu sofrimento em uma abordagem holística, para além de uma dimensão exclusivamente orgânica.

Nessa perspectiva, haja vista o lugar hegemônico conferido à prole (BORSA; NUNES,
A coleta dos dados demonstrou que da totalidade de centros reprodutivos existentes no país, a grande maioria localiza-se nas regiões Sul e Sudeste, como observado na Figura 1. Pôde-se perceber que São Paulo é o estado brasileiro com a maior quantidade de clínicas voltadas à realização da reprodução humana assistida. Além disso, no estado em questão encontra-se a maior quantidade de recursos utilizados na produção e no armazenamento de embriões e células reprodutivas.
CENTROS REPRODUTIVOS NO BRASIL

$>18$
$>10$
$06-08$
$01-04$
0
2011), a constatação da impossibilidade de tê-los comumente desencadeia um processo de grande sofrimento psíquico às famílias. Além disso, estudos empíricos verificam a existência de desgaste relacional em um período prolongado acima de 4 anos de tratamento sem obtenção de sucesso, ou seja, a não ocorrência da gravidez. São evidenciados, também, o aumento de ansiedade e sintomas depressivos em mulheres após duas semanas do primeiro ciclo de fertilização in vitro (FIV) sem sucesso e ao longo de até um ano após a falha de um ciclo de FIV. O abandono do tratamento pode ocorrer desde o primeiro ciclo de FIV sem êxito, e a incidência tende a aumentar a cada ciclo sem sucesso. Estas nuances, por sua vez, ilustram a complexidade dos contextos social, econômico, emocional e relacional dos casais que vivenciam o problema de infertilidade e a sua jornada diante dos tratamentos de reprodução assistida (MARQUES; 
MORAIS, 2018).

Nesse viés, a popularização das técnicas existentes, bem como o interesse populacional em remediar a infertilidade humana, proporcionou um aumento exponencial na procura pela reprodução humana assistida. Assim, como evidenciado em Figura 2 , é possível observar que a RHA foi significativamente mais requerida em estados de maior desenvolvimento econômico. Neste panorama, a maior oferta de tecnologias e oportunidades nas regiões mais desenvolvidades do país, induz a migração de inidivíduos que buscam o acesso descomplicado à tratamentos não existentes em suas localidades residenciais (SANTOS; PEREIRA; DELDUQUE, 2019).

Figura 2. Percentual estadual de oócitos produzidos e fertilizações humanas realizadas, reguladas pelo SisEmbrio no ano de 2016.

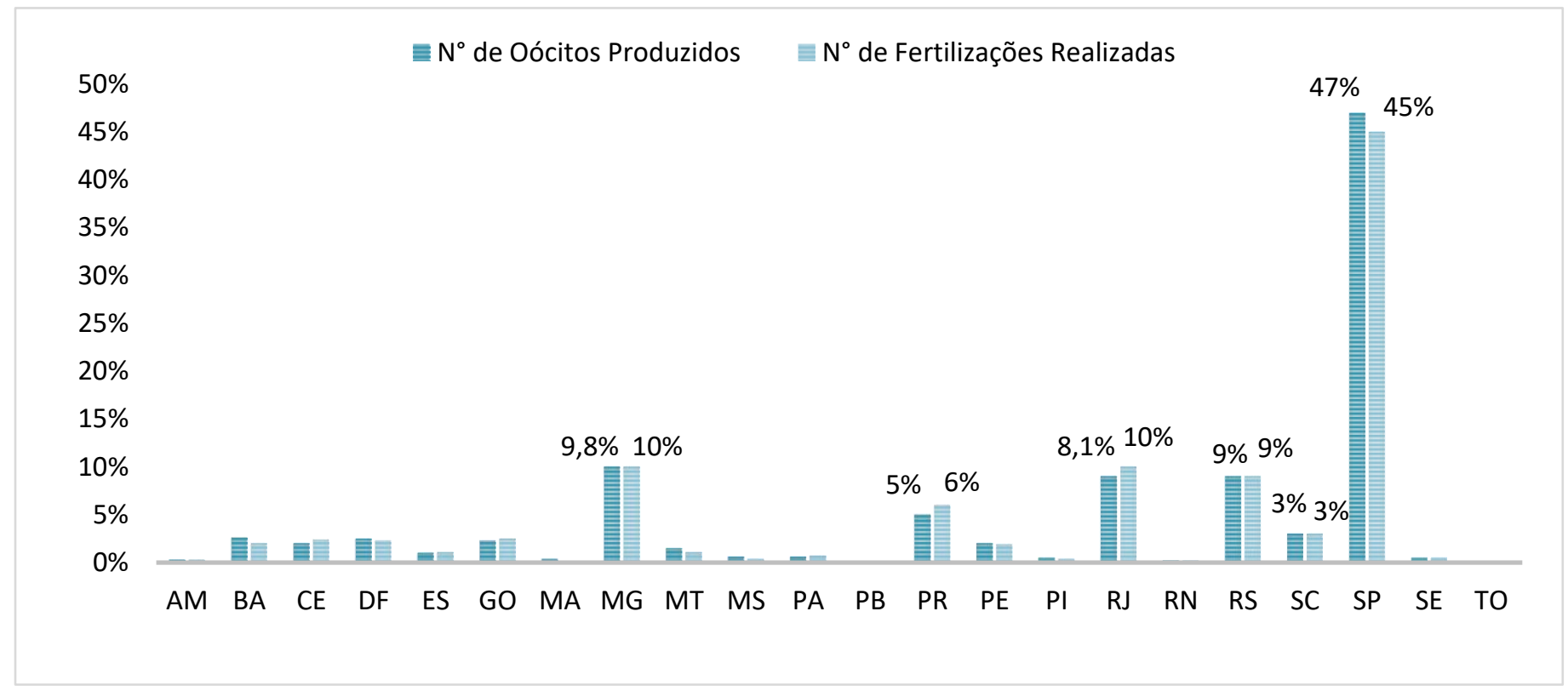

Fonte: (SISEMBRIO, 2016).

Seguindo este princípio, será analisada a evolução do material embrionário desenvolvido nas instituições brasileias. Para isso, serão apresentados e discutidos o percentual de células embrionárias congeladas em cada região do Brasil (Figura 3) e o percentual dos embriões descartados por estado no Brasil (Figura 4). 
Figura 3. Percentual de células embrionárias congeladas em cada região do Brasil no ano de 2016, de acordo com o SisEmbrio.

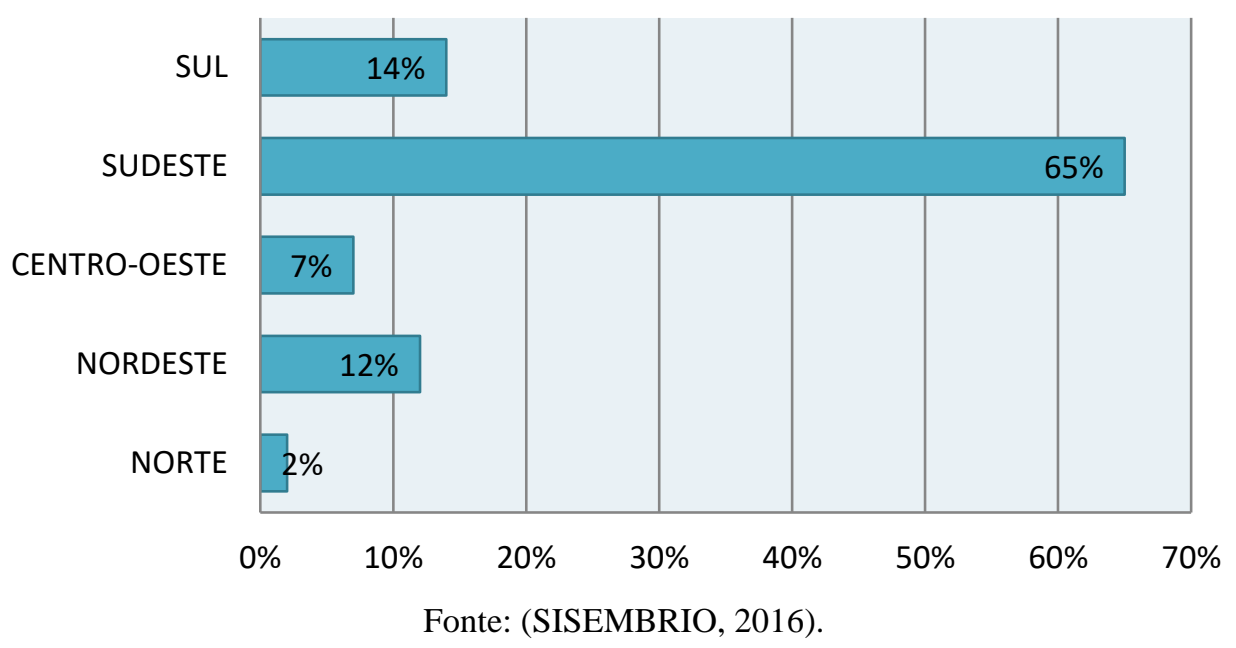

Como observado na Figura 4, a maior quantidade de embriões descartados pertence ao estado de São Paulo, localizado na região Sudeste. Correlacionando a Figura 3 com a Figura 4, pode-se entender que a quantidade de células embrionárias descartadas é diretamente proporcional a quantidade de células embrionárias congeladas.

Figura 4. Percentual dos embriões descartados por estado no Brasil no ano de 2016, de acordo com o SisEmbrio.

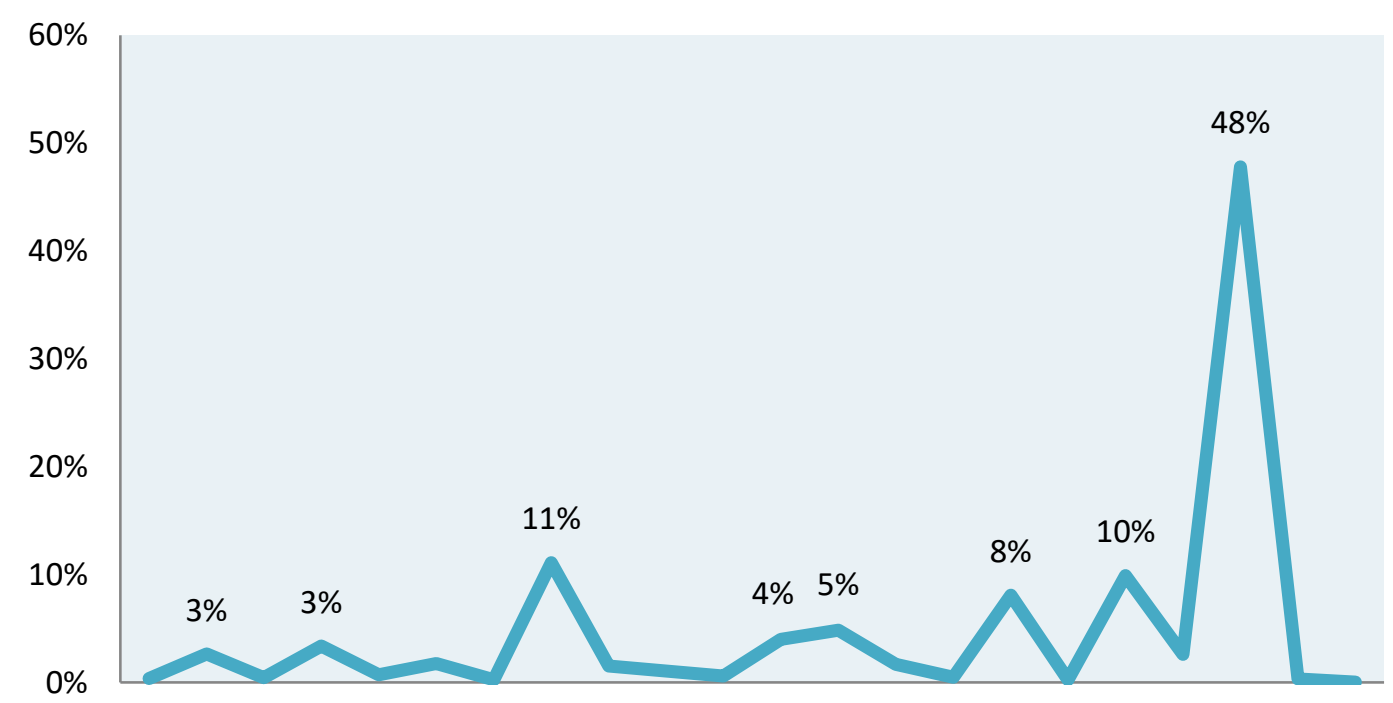

AM BA CE DF ES GO MAMG MT MS PA PB PR PE PI RJ RN RS SC SP SE TO

Fonte: (SISEMBRIO, 2016).

Pode-se observar que mais da metade dos embriões congelados no Brasil se encontram na região Sudeste. Essa, por sua vez, concentra alguns dos maiores centros de pesquisa e desenvolvimento do país, o que atrai indivíduos de outras regiões interessados no serviço (LEITE, 2019). Dessa forma, entende-se que, por possuir uma maior população e um maior nível tecnológico, a região Sudeste compreende a maioria dos procedimentos realizados e dos embriões armazenados. Outrossim, em uma análise quanto o material 
embrionário armazenado nas instituições, como denotado na Figura 4, a maior quantidade de embriões descartados pertence ao estado de São Paulo, localizado na região Sudeste. Logo, correlacionando a Figura 3 com a Figura 4, pode-se entender que a quantidade de células embrionárias descartadas é diretamente proporcional a quantidade de células embrionárias congeladas.

As técnicas de criopreservação possibilitam que os gametas, embriões e tecidos gonádicos sejam armazenados em baixas temperaturas por um período indeterminado. Regulamenta-se no Brasil que as células reprodutivas podem ser armazenadas por até três anos sendo descartadas após esse período em casos de abandono ou escolha dos pacientes (BRASIL, 2017b). Um documento específico que trata da destinação do material biológico em casos de divórcio e morte deve ser assinado por ambos os doadores. Destaca-se que os embriões remanescentes só podem ser encaminhados para pesquisa científica mediante o consentimento dos doadores (BENTO et al., 2019; GASDA, 2015).

De acordo com documentos do Ministério da Saúde, objetivando a diminuição do número de gestações múltiplas, proíbe-se a implantação de todos os embriões produzidos em laboratório na receptora (BRASIL,2017b). Vale salientar que a quantidade de embriões transferidos é diferente entre mulheres de menor idade e de idade mais avançada. Para mulheres em idade fértil (até 35 anos), determina-se a implantação de apenas 2 embriões, enquanto mulheres com idade entre 36 e 49 anos podem receber até 3 embriões por procedimento. Por fim, para mulheres com idade maior que 40 anos se determina a implantação de 4 embriões que é a quantidade máxima permitida. Os embriões excedentes geralmente são criopreservados para uso posterior (FRANCO; SANTO MENDES, 2019).

Consoante Frank et al. (2015) os embriões e células reprodutivas excedentes podem ser criopreservados para uso posterior ou podem ser destinados à pesquisa com células-tronco embrionárias. O Conselho Federal de Medicina regulamenta, através da resolução $n^{\circ} 2.168 / 2017$, a respeito das normas éticas para realização das técnicas de reprodução assistida, bem como sobre os parâmetros para armazenamento e descarte dos materiais reprodutivos. Institui-se, portanto, que a fecundação de oócitos humanos apenas pode ser realizada com o propósito exclusivo de reprodução. As técnicas utilizadas não podem ser aplicadas com a finalidade de selecionar sexo ou outras características biológicas do possível embrião (BRASIL, 2017b, SILVA et al., 2016).

Neste panorama, também surge uma das maiores adversidades da medicina reprodutiva atual: a necessidade de preservação da fertilidade feminina e masculina. No âmbito em questão, destaca-se a preocupação com a saúde reprodutiva de pacientes que serão submetidos a tratamentos oncológicos e a terapias gonadotóxicas. Ambos são procedimentos substancialmente tóxicos para o organismo humano e podem levar a graves complicações do sistema reprodutor (MOURA et al., 2016; SANTOS, 2017). Conforme as diretrizes da Sociedade Americana de Oncologia Clínica, os médicos oncologistas devem orientar os pacientes sobre as alternativas de preservação à fertilidade e entre elas a mais indicada é a metodologia da criopreservação. Os tipos de material biológico a serem criopreservados, variam de acordo com o sexo e a idade dos pacientes em questão (OKTAY et al., 2018).

Para os homens e mulheres em idade reprodutiva são grandes as opções de material para congelamento. Pode-se criopreservar tanto os tecidos germinativos, como também as células reprodutivas isoladas e até o proprio embrião já fecundado. Já na preservação da fertilidade de crianças, o único material que poder ser congelado é o tecido germinativo. Este posteriormante necessita passar pela maturação in vitro,entretanto, tal procedimento ainda possui caráter experimental (STRAUBE ; MELAMED, 2015).

Além dos pacientes que realizam quimioterapia e outros tratamentos oncológicos, a criopreservação de gametas e embriões ainda pode ser realizada para inidivíduos transexuais que passam por tratamentos hormonais e cirúrgicos agressivos que implicam na perca significativa do seu potencial de fertilidade (OLIVEIRA ; OLIVEIRA JÚNIOR ; ANDRADE, 2017).

\section{CONCLUSÃO}

A temática abordada possui alta relevância pela possibilidade de remediar diversas enfermidades reprodutivas e viabilizar a preservação de gametas e embriões, garantindo assim que indivíduos que não possuíam tal perspectiva, possam gerar descendentes. Diante do presente exposto, constata-se que houve um aumento significativo, entre os anos de 2015 e 2017, na procura pela realização da Reprodução Humana Assistida. Visto que, com o avanço dos meios de comunicação, ocorreu a popularização das metodologias existentes, proporcionando assim, um maior interesse populacional por procedimentos médicos capazes de remediar a infertilidade humana. 
Por conseguinte, denota-se que a Reprodução Humana Assistida engloba um conjunto de técnicas de alto custo e difícil acesso para pacientes de baixo poder aquisitivo. Verificou-se, além disso, que nas regiões Sul e Sudeste, foram executados mais procedimentos associados a RHA. Tais localidades possuem o índice econômico compatível com a realização das metodologias e, por isso, assumem a liderança na realização dos procedimentos. Paralelamente, elas são consideradas polos de pesquisa e inovação, tornando-se assim alvos constantes da migração de indivíduos que almejam constituir uma família pelos métodos de RHA.

\section{REFERÊNCIAS}

BATISTA， L.A.T.; BRETONES， W.H.D.; DE ALMEIDA, R.J. O impacto da infertilidade: narrativas de mulheres com sucessivas negativas pelo tratamento de reprodução assistida. Reprodução \& Climatério, v. 31, n. 3, p. 121-127, 2016.

BENTO, L.A. et al., Bioética e reprodução humana assistida homóloga post-mortem. Revista Uningá, v. 56, n. 2, p. 215-227, 2019.

BORSA, J.C.; NUNES, M.N.T. Aspectos psicossociais da parentalidade: $O$ papel de homens e mulheres na família nuclear. Psicol. Argum, Curitiba, v. 64, n. 26, p.31-39, jan. 2011.

BRASIL, AGÊNCIA DE VIGILÂNCIA SANITÁRIA. SisEmbrio $-\mathbf{1 0}^{\circ}$ Relatório do Sistema Nacional da Produção de Embriões, 2017b. Disponível em < http://portal.anvisa.gov.br/documents/33840/2817584/ $10 \% \mathrm{C} 2 \% \mathrm{BA}+$ Relat\%C3\%B3rio+do+Sistema+Nacion al+de+Produ $\%$ C3\%A7\%C3\%A3o+de+Embri\%C3\%B 5es+-+SisEmbrio/1121df4c-ab05-47e9-bae0-

8dc283f36fbc > Acesso em: 18 de março. 2018.

BRASIL. Resolução CFM 2.168, de 10 de novembro de 2017. Brasília, 2017a. Disponível em: < https://sistemas. cfm.org. br/normas/visualizar/resoluções/BR/2017/2168 >. Acesso em: 16 de outubro de 2018.

CORRÊA, M.C.D. V.; LOYOLA, M. A. Tecnologias de reprodução assistida no Brasil: opções para ampliar o acesso. Physis-Revista de Saúde Coletiva, v. 25, n. 3, p. 753-777, 2015.
FRANCO, M.L.; SANTO MENDES, N.B.E. Bioética Aplicada a Reprodução Humana Assistida. Biológica-Caderno do Curso de Ciências Biológicas, v. 1, n. 1, 2019.

GASDA, E.E. Criopreservação de embriões humanos no contexto da saúde sexual e reprodutiva. Revista Pistis \& Praxis: Teologia e Pastoral, v. 7, n. 3, p 635661. 2015.

LEITE, T.H. Análise crítica sobre a evolução das normas éticas para a utilização das técnicas de reprodução assistida no Brasil. Ciência \& Saúde Coletiva, v. 24, p. 917-928, 2019.

LIMA, A. P. W.; LOURENÇO, J. W. Infertilidade humana: comentando suas causas e consequências. Revista Saúde e Desenvolvimento, v. 10, n. 5, p. 110-124, 2016.

MACHADO C.S. Bioética na reprodução humana assistida: os impactos éticos e emocionais no destino de embriões excedentários, p. 215, 2016. Dissertação (Mestrado em Serviço Social) - Faculdade Ciências Humanas e Sociais, Universidade Estadual Paulista "Júlio de Mesquita Filho", 2016.

MARCIANO, R. et al., Considerações éticas e bioéticas em reprodução humana assistida. Revista Bioética Cremego, v. 1, n. 01, p. 9-18, 2019.

MARQUES, P. P.; MORAIS, N.A. A vivência de casais inférteis diante de tentativas inexitosas de reprodução assistida. Avances En Psicología Latinoamericana, v. 36, n. 2, p. 299-314, 2018. 
MATIAS, E.A. Reprodução humana assistida e adoção infantil no Brasil: uma análise das ações do Estado na perspectiva da bioética crítica. 2018, p. 23. Dissertação (Mestrado em Bioética) - Universidade de Brasília, Brasília, 2018.

MESQUITA, M.O. et al. Acesso e permanência das usuárias do Sistema Único de Saúde no Programa de Reprodução Assistida de um hospital universitário, com ênfase na variável raça/cor. Revista Reprodução e Climatério, v. 21, n. 3, p. 151-158. 2016.

MOURA, B.V.C.S. et al. Métodos de preservação de fertilidade: revisão de literatura. Brazilian Journal of Surgery and Clinical Research, v. 13, n.3, p. 56-64, 2016.

NETO, M.L.A.S. et al. Debatendo a procriação medicamente assistida. 2018.

OKTAY, K. et al. Fertility Preservation in Patients with Cancer: ASCO Clinical Practice Guideline Update. Journal of Clinical Oncology, v. 36, n. 19, p. 1994-2001, 2018.

OLIVEIRA JÚNIOR, E.Q.; OLIVEIRA, P.Q.; ANDRADE G.O. Cirurgia Transexual: realidade médica, legal e social. Revista Jurídica da Escola Superior do Ministério Público de São Paulo, v. 10, n. 2, p. 115-130, 2017.

OLIVEIRA, A.E.F. et al. Os aspectos jurídicos da reprodução humana assistida post mortem. Revista de trabalhos acadêmicos-Universo Belo Horizonte, v. 1, n. 3, 2018 .

SANTOS, A.M.J. Métodos de preservação da fertilidade em doentes submetidas a terapêuticas gonadotóxicas. 2017, p. 34. Tese (Mestrado integrado em Medicina) - Clínica Universitária de Obstetrícia e Ginecologia, Faculdade de Medicina de Lisboa, Lisboa, 2017.

SANTOS, A.O.; PEREIRA, A.G.D.; DELDUQUE, M.C. Reprodução humana assistida: regulamentação no Brasil e em Portugal. Revista Jurídica da Escola Superior do Ministério Público de São Paulo, v. 16, n. 2, 2019.

SANTOS, V. F. D. Reprodução humana assistida em casais homoafetivos: reflexões acerca da gestação por substituição na realidade brasileira contemporânea, 2019, p.8. Monografia (Graduação em Direito) - UCSAL, Universidade Católica do Salvador, Salvador, 2019.

SILVA, J.D. A reprodução humana assistida e ausência de uma lei específica no ordenamento jurídico brasileiro. 2016, p. 76. Monografia (Graduação em Direito) - Centro de Educação, Faculdades integradas Santa Cruz de Curitiba, Curitiba, 2016.

STRAUBE, K.M.; MELAMED, R.M. Temas Contemporâneos de Psicologia em Reprodução Humana Assistida: A infertilidade em seu espectro psicoemocional. Editora Livrus, 2015. 